\title{
THE CHILD-LANGMUIR LIMIT FOR SEMICONDUCTORS: A NUMERICAL VALIDATION
}

\author{
María-José Cáceres ${ }^{1}$, José-Antonio Carrillo ${ }^{1}$ and Pierre Degond ${ }^{2}$
}

\begin{abstract}
The Boltzmann-Poisson system modeling the electron flow in semiconductors is used to discuss the validity of the Child-Langmuir asymptotics. The scattering kernel is approximated by a simple relaxation time operator. The Child-Langmuir limit gives an approximation of the currentvoltage characteristic curves by means of a scaling procedure in which the ballistic velocity is much larger that the thermal one. We discuss the validity of the Child-Langmuir regime by performing detailed numerical comparisons between the simulation of the Boltzmann-Poisson system and the Child-Langmuir equations in test problems.
\end{abstract}

Mathematics Subject Classification. 35L65, 65M99, 82D37.

Received: May 3, 2002. Revised: July 10, 2002.

\section{INTRODUCTION}

We consider the 1-D relaxation-time kinetic system for semiconductors [16],

$$
\frac{\partial F}{\partial t}+V \frac{\partial F}{\partial X}-\frac{e}{m} E(t, X) \frac{\partial F}{\partial V}=\frac{1}{\tau}\left(M_{T}(V) \rho(F)-F\right)
$$

$F=F(t, X, V)$ is the density function for an electron at position $X \in[0, L]$ and velocity $V \in \mathbb{R}$ at time $t \geq 0$, where $L$ is the device channel length. $e$ and $m$ are the unit charge and effective electron mass, respectively. The electric field $E=E(t, X)$ is self-consistently produced by the motion of electrons in a fixed ion background with density $C(X)$, called doping profile. $E$ is determined by the Poisson equation

$$
\epsilon_{0} \frac{\mathrm{d}^{2} \phi}{\mathrm{d} X^{2}}=e(\rho(F)-C(X)), \quad E(t, X)=-\frac{\mathrm{d} \phi}{\mathrm{d} X},
$$

with $\epsilon_{0}$ the permitivity of the material and $\rho(F)(t, X), J(t, X)$ are, respectively, the particle density and flux of the electrons, i.e., the first two moments of the distribution $F(t, X, V)$. The relaxation time $\tau$ is assumed

\footnotetext{
Keywords and phrases. Boltzmann-Poisson system, Child-Langmuir limit, WENO schemes, semiconductor devices.

1 Departamento de Matemática Aplicada, Universidad de Granada, 18071 Granada, Spain.

e-mail: caceresg@ugr.es, carrillo@ugr.es

2 MIP, UMR CNRS 5640, Université Paul Sabatier, 118, route de Narbonne, 31062 Toulouse Cedex, France.

e-mail: degond@mip.ups-tlse.fr
} 
constant and related to standard values of the mobility $\mu$ by $\mu=\frac{e}{m} \tau$. $T$ is the lattice temperature, $M_{T}$ is the equilibrium maxwellian associated with this temperature

$$
M_{T}(V)=\frac{1}{\sqrt{\frac{2 \pi k_{\mathrm{B}} T}{m}}} \exp \left(-\frac{m V^{2}}{2 k_{\mathrm{B}} T}\right)
$$

and $k_{\mathrm{B}}$ is the Boltzmann constant.

The Child-Langmuir law is originally concerned with the description of electron flow in a vacuum diode when space charge phenomena are important, and specifically when the energy of the particles emitted at the cathode is small compared with the applied voltage. Child, Langmuir and Compton performed the analysis of this problem in $[15,1930]$ in the case of a one-dimensional plane vacuum diode and proved the Child-Langmuir law: the current which flows through the diode, under these hypotheses, depends not on the number of extracted electrons but only on the applied bias and the geometry of the diode. 60 years later, at 1991, this law was obtained, in [12], as a limit solution of a perturbation problem for the one-dimensional Vlasov-Poisson system. The Child-Langmuir law has many diverse applications, and one of them is to semiconductors [1,4,7,9], and we will focus on this application. Section 2 is devoted to an overview of the Child-Langmuir (CL) limit in a unipolar $\mathrm{N}^{+}-\mathrm{N}^{-}-\mathrm{N}^{+}$diode. Ballistic transport in semiconductors have been studied for practical purposes in $[2,18,19]$.

The aim of this paper is to numerically validate the CL asymptotic limit for the 1-D relaxation time kinetic system. In order to do so, we show numerical simulations for the CL limit and we compare these numerical results with deterministic computations for the kinetic system. These deterministic numerical simulations were given in [11], where the authors analysed and compared several classical approximations to the Boltzmann-Poisson system (hydrodynamics, drift-diffusion systems), and were validated with Monte Carlo (DSMC) results for a silicon diode in [10]. These simulations are based on the use of Weighted Essentially Non-Oscillatory (WENO) schemes $[14,17]$. We will report simulations for the same device in two different materials: Si and GaAs. We observe numerically two facts: the results get better as the applied bias gets higher or as the lattice temperature gets lower. These results agree with the assumptions underlying the Child-Langmuir law. Moreover, we perform comparisons of the distribution function of the kinetic system with the predicted distribution function in the CL limit [5]. Also, based on this asymptotic formula we compare moments of the kinetic solution and the CL limiting solution.

We structure the paper as follows: Section 2 gives a brief overview for the Child-Langmuir limit. In Section 3 we focus on the numerical comparisons of the CL limit, with the solution of the full Boltzmann equation. Finally, we draw conclusions in the last section.

\section{An OVERVIEW OF THE CHILD-LANGMUIR LIMIT}

Let us consider a unipolar $\mathrm{N}^{+}-\mathrm{N}^{-}-\mathrm{N}^{+}$device, which means two highly doped $\mathrm{N}^{+}$regions on each side of a lowly doped $\mathrm{N}^{-}$region. In the $\mathrm{N}^{+}$zone, the electrons are at a thermodynamic quasi-equilibrium and have a density approximately equal to $\mathrm{N}^{+}$, i.e., $F(X, V)=N^{+} M_{T}\left(V-V_{0}\right)$ with a small mean velocity $V_{0}$, and thus the electrostatic potential is nearly constant in the $\mathrm{N}^{+}$zones. At the interface of the $\mathrm{N}^{+}$and the $\mathrm{N}^{-}$region, the doping density falls down dramatically causing an excess of negative charge in the $\mathrm{N}^{-}$region near the interface, giving rise to the so-called built-in potential.

The rest of this section is aimed at a brief description of the CL asymptotic limit. The reader can find more details in $[1,3,4,6,7]$. A convergence proof of the CL limit for the relaxation time operator can be found in $[4,6]$. Here, we focus on its numerical performance and validation with respect to the simulation of the Boltzmann-Poisson system. The CL limit describes the stationary distribution function in the channel region, replacing the $\mathrm{N}^{+}$zones by appropriate boundary conditions at the $\mathrm{N}^{ \pm}-\mathrm{N}^{\mp}$ junctions. Therefore, we deal only with the channel or $\mathrm{N}^{-}$region represented by the interval $[0, L]$. In this way, the junctions are located at $X=0$, 
for the source side, and $X=L$, for the drain side. Since we are interested in steady state results, we consider the stationary system associated with (1.1), namely,

$$
V \frac{\partial F}{\partial X}+\frac{e}{m} \frac{\mathrm{d} \phi}{\mathrm{d} X} \frac{\partial F}{\partial V}=\frac{1}{\tau}\left(M_{T}(V) \rho(F)-F\right)
$$

where $\frac{\mathrm{d}^{2} \phi}{\mathrm{d} X^{2}}=\frac{e}{\epsilon_{0}}(\rho(F)-C(X))$. In order to take into account the $\mathrm{N}^{+}$zones, we assume the following boundary conditions: injection of electrons according to a certain distribution function, $G(V)$, at the $\mathrm{N}^{+}-\mathrm{N}^{-}$interface on the source side, and absence of electron injection at the $\mathrm{N}^{-}-\mathrm{N}^{+}$interface on the drain side, i.e.,

$$
F(0, V)=G(V) \quad V>0, \quad F(L, V)=0 \quad V<0 .
$$

$G$ is assumed to coincide with the thermodynamical equilibrium distribution function of the electrons in the source region: $G(V)=N^{+} M_{T}(V)$. We consider the diode with an applied bias and thus,

$$
\phi(0)=0, \quad \phi(L)=\phi_{L}>0 .
$$

In the CL regime, the thermal emission energy is small compared with the applied bias, while the injection current remains finite [12], $m V_{\mathrm{th}}^{2} / 2 \ll e \phi_{L}$. Or, equivalently, the mean velocity of the injected electrons at the source side is small compared with their velocity when they reach the drain side, $V_{\mathrm{th}} \ll V_{L}$, where $V_{\mathrm{th}}=\sqrt{k_{\mathrm{B}} T / m}$ is the thermal velocity, and $V_{L}=\sqrt{2 e \phi_{L} / m}$ is the ballistic velocity at the end of the channel. Under this hypothesis, we can introduce the following scaling parameter

$$
\epsilon=\frac{V_{\mathrm{th}}}{V_{L}}=\sqrt{\frac{k_{\mathrm{B}} T}{2 e \phi_{L}}} \ll 1 .
$$

We shall use $L, V_{L}$ and $\phi_{L}$ as characteristic length, velocity and potential. We introduce auxiliary units of density $N_{L}$, current density $J_{L}$ and distribution function $F_{L}$, according to

$$
N_{L}=\frac{\epsilon_{0} \phi_{L}}{e L^{2}}, \quad F_{L}=\frac{N_{L}}{V_{L}}, \quad J_{L}=e N_{L} V_{L}, \quad \tau_{L}=\frac{L}{V_{L}},
$$

and use the following scaling:

$$
\left\{\begin{array}{l}
X=L x, \quad V=V_{L} v, \quad \phi=\phi_{L} \varphi^{\epsilon}, \quad \tau=\tau_{L} \tilde{\tau} \\
\rho(F)=N_{L} n^{\epsilon}, \quad J=-J_{L} j^{\epsilon}, \quad F=F_{L} f^{\epsilon}, \quad C(X)=N_{L} n_{\mathrm{D}} .
\end{array}\right.
$$

This scaling means that the relaxation time has the same order of magnitude as the electron transit time in the $\mathrm{N}^{-}$zone. Furthermore, we introduce a dimensionless profile $g(v)$ and express $G(V)$ according to

$$
F_{L}^{-1} G(V)=\frac{1}{\epsilon^{2}} g\left(\frac{V}{V_{\mathrm{th}}}\right)=\frac{1}{\epsilon^{2}} g\left(\frac{v}{\epsilon}\right),
$$

which means that $V_{\text {th }}$ is the characteristic velocity associated with $G$, and the factor $\epsilon^{2}$ ensures that the injected current $J_{G}$ remains independent of $\epsilon, J_{G}=J_{L} j_{g}$, where $j_{g}$ is the dimensionless injected current. On other hand, (2.6) gives the following relation $\frac{N^{+}}{N_{L}}=\frac{1}{\epsilon}$. This is coherent with the hypothesis that the density in the $\mathrm{N}^{+}$zone is large. 
After the change of scales (2.5) and passing formally to the limit $\epsilon \rightarrow 0$ (see [5,6] for details), we obtain

$$
\begin{gathered}
v \frac{\partial f}{\partial x}+\frac{1}{2} \frac{\mathrm{d} \varphi}{\mathrm{d} x} \frac{\partial f}{\partial v}=\frac{1}{\tilde{\tau}}(n \delta(v)-f), \\
\frac{\mathrm{d}^{2} \varphi}{\mathrm{d} x^{2}}=n(x)-n_{\mathrm{D}}, \quad x \in[0,1], \quad n(x)=\int_{-\infty}^{+\infty} f(x, v) \mathrm{d} v \\
\varphi(0)=0, \quad \varphi(1)=1,
\end{gathered}
$$

where $\delta(v)$ is the delta function centered at 0 . An expression of the distribution function $f(x, v)$ can be given, taking into account the trajectories of electrons,

$$
f(x, v)=n_{1}(x) \delta[v-\sqrt{\varphi(x)}]+\int_{0}^{x} \bar{n}_{2}(x, y) \delta[v-\sqrt{\varphi(x)-\varphi(y)}] \mathrm{d} y
$$

where $n_{1}(x)$ is the density of particles carried by the principal characteristics and $\bar{n}_{2}(x, y) \mathrm{d} y$ is the density of particles carried by the bundle of characteristics issued between the points $(y, 0)$ and $(y+\mathrm{d} y, 0) . n_{1}(x)$ and $\bar{n}_{2}(x, y)$ verify a system of integro-differential equations (see [6]).

As mentioned above, the potential barrier lying at the $\mathrm{N}^{+}-\mathrm{N}^{-}$junction is large in the semiconductor case. In the CL asymptotics the potential barrier vanishes.

The condition (2.6) does not lead to large enough densities to account for the experimental doping densities. Then, in order to have a potential barrier of order 1, it is necessary to model the built-in potential. This modeling can be seen in [9]. The idea is to rescale the injection condition, so as to produce a finite built-in potential barrier at the source junction, and a potential drop at the drain junction. The resulting limit problem is related with the above limit problem by a translation of the potential by the value $-\beta$, where $\beta$ is the potential barrier.

Therefore, in the numerical simulations shown in next section we work with the simpler law (2.6), but we will take into account the influence of the built-in potential.

At equilibrium the built-in potential is given by the relation $V_{\mathrm{bi}}=U_{\mathrm{th}} \log \frac{N^{+}}{N^{-}}$, where $U_{\mathrm{th}}=k_{\mathrm{B}} T / e$ is the thermal voltage. Therefore, the scaled built-in potential is $\beta=V_{\mathrm{bi}} / \phi_{L}$. This is the value of $\beta$ used in the numerical simulations. We refer to [9] for the modeling of the built-in potential away of equilibrium.

Remark 2.1. Let us point out that the scaling (2.5) coincides with the ballistic scaling introduced in [11]. For more details we refer to [11], where the reader can find an overview of the different scaling limits for the relaxation system. In this reference the relaxation time was considered as a function of the electric field producing a finite saturation speed. In our case the relaxation time is constant and thus, the mean velocity as a function of the electric field in the homogeneous case has no upper limit.

\section{Numerical Simulation}

In this section, we focus on the aim of this paper, i.e., to numerically validate the CL asymptotic limit for the 1-D relaxation time kinetic system $(1.1,1.2)$. Let us compare numerical simulations for the CL limit with deterministic computations for the kinetic system. The numerical study consists in comparison of the following quantities: current-voltage characteristic curves, potentials, densities, distribution functions, velocities, energies, temperatures and third moments, for differents applied biases, materials ( $\mathrm{Si}$ and GaAs) and temperatures (77 K and $300 \mathrm{~K})$. 
TABLE 1. Values of $\epsilon$.

\begin{tabular}{||l|l|l|l||}
\hline$\phi_{L}$ & 0.5 & 1 & 2 \\
\hline$\epsilon$ at $77 \mathrm{~K}$ & 0.0815 & 0.0576 & 0.0407 \\
\hline$\epsilon$ at $300 \mathrm{~K}$ & 0.1608 & 0.1137 & 0.0804 \\
\hline
\end{tabular}

TABLE 2. Units.

\begin{tabular}{||l|l||}
\hline$x: \mu \mathrm{m}$ & Potential: $\mathrm{V}$ \\
\hline Temperature: $\mathrm{K}$ & Velocity: $10^{7} \mathrm{~cm} \mathrm{~s}^{-1}$ \\
\hline Density: $\mathrm{cm}^{-3}$ & Current: $10^{2} \mathrm{~A} \mathrm{~cm}^{-2}$ \\
\hline Energy: $\mathrm{eV}$ & Third moment: $10^{21} \mathrm{~s}^{-3}$ \\
\hline Mean temperature: $\mathrm{eV}$ & Normalized distribution function: $10^{-7} \mathrm{~s} \mathrm{~cm}^{-1}$ \\
\hline
\end{tabular}

TABle 3. Parameters for Silicon.

\begin{tabular}{||l|l||}
\hline$m=0.26 \times 0.9109 \cdot 10^{-30} \mathrm{~kg}$ & $\mu=1323 \frac{\mathrm{cm}^{2}}{\mathrm{Vs}}$ \\
\hline$\epsilon_{0}=11.7 \times 8.85419 \cdot 10^{-14} \mathrm{~F} \mathrm{~cm}^{-1}$ & $\tau \simeq 1.956 \times 10^{-13} \mathrm{~s}$ \\
\hline
\end{tabular}

The method for deterministic simulations was given in [11]. Therefore we only point out the main used tools. WENO finite-difference schemes [14,17], which are designed for hyperbolic conservation laws or other problems containing either discontinuous solutions or solutions with sharp gradients, are used to approximate the spatial and velocity derivatives. They are particularly well suited to sharp interfaces on the density profile. Then, we are able to solve the time dependent problem with an explicit Runge-Kutta method due to the stability of the WENO approximation of the derivatives. We run the simulations until the time variation of the density and current stabilize and then decide that a steady state has been achieved. The self-consistent potential $\phi$ is updated at each time step by solving the Poisson equation (1.2).

The numerical scheme, for approximating the potential and the current, for the CL was given by Ben Abdallah in [5]. It is based on a shooting method for solving the boundary value problem $(2.8,2.9)$.

The numerical approximation for $f$ is given by the expression (2.10), where we have to estimate $\delta(v)$. We will substitute $\delta(v)$ by $\frac{1}{\lambda} M_{0}\left(\frac{v}{\lambda}\right)$ with $\lambda$ small enough, and the resulting distribution given by (2.10) will be denoted by $h^{\lambda}$. Note that $\lambda$ should be chosen equal to $\epsilon$ given by the relation (2.4) since $\epsilon$ is small enough in applications (see Tab. 1).

For the numerical simulations of the CL asymptotic limit we deal only with the channel region $\mathrm{N}^{-}$, while the deterministic simulation of the Boltzmann-Poisson system is performed in the whole device, $\mathrm{N}^{+}-\mathrm{N}^{-}-\mathrm{N}^{+}$. We develop these simulations for the same device with two different materials: Si and GaAs. We cut the velocity space, considering $[-a, a]$ large enough, such that out of this interval the distribution function vanishes. We set $a=3.5 \times 10^{8} \mathrm{~cm} \mathrm{~s}^{-1}$, which is in the same scale as $V_{L}$ and $V_{\mathrm{th}}$, and both velocities are smaller than $a$. Table 2 shows the units will be used in the following.

\subsection{Silicon and GaAs device models}

For our simulations we consider a one dimensional $\mathrm{N}^{+}-\mathrm{N}^{-}-\mathrm{N}^{+}$structure of channel length $L=0.4 \mu \mathrm{m}$, where the domain of the device is $\Omega=[-0.1 \mu \mathrm{m}, 0.5 \mu \mathrm{m}]$, for two different materials; Si and GaAs. And they are performed for applied biases $\phi_{L}$ increasing from $0.1 \mathrm{~V}$ to $2 \mathrm{~V}$ with uniform increments of $0.1 \mathrm{~V}$. 
TABle 4. Parameters for GaAs.

\begin{tabular}{||l|l||}
\hline$m=0.065 \times 0.9109 \cdot 10^{-30} \mathrm{~kg}$ & $\mu=34903.4 \frac{\mathrm{cm}^{2}}{\mathrm{Vs}}$ \\
\hline$\epsilon_{0}=13.2 \times 8.85419 \cdot 10^{-14} \mathrm{~F} \mathrm{~cm}^{-1}$ & $\tau=12.9 \cdot 10^{-13} \mathrm{~s}$ \\
\hline
\end{tabular}
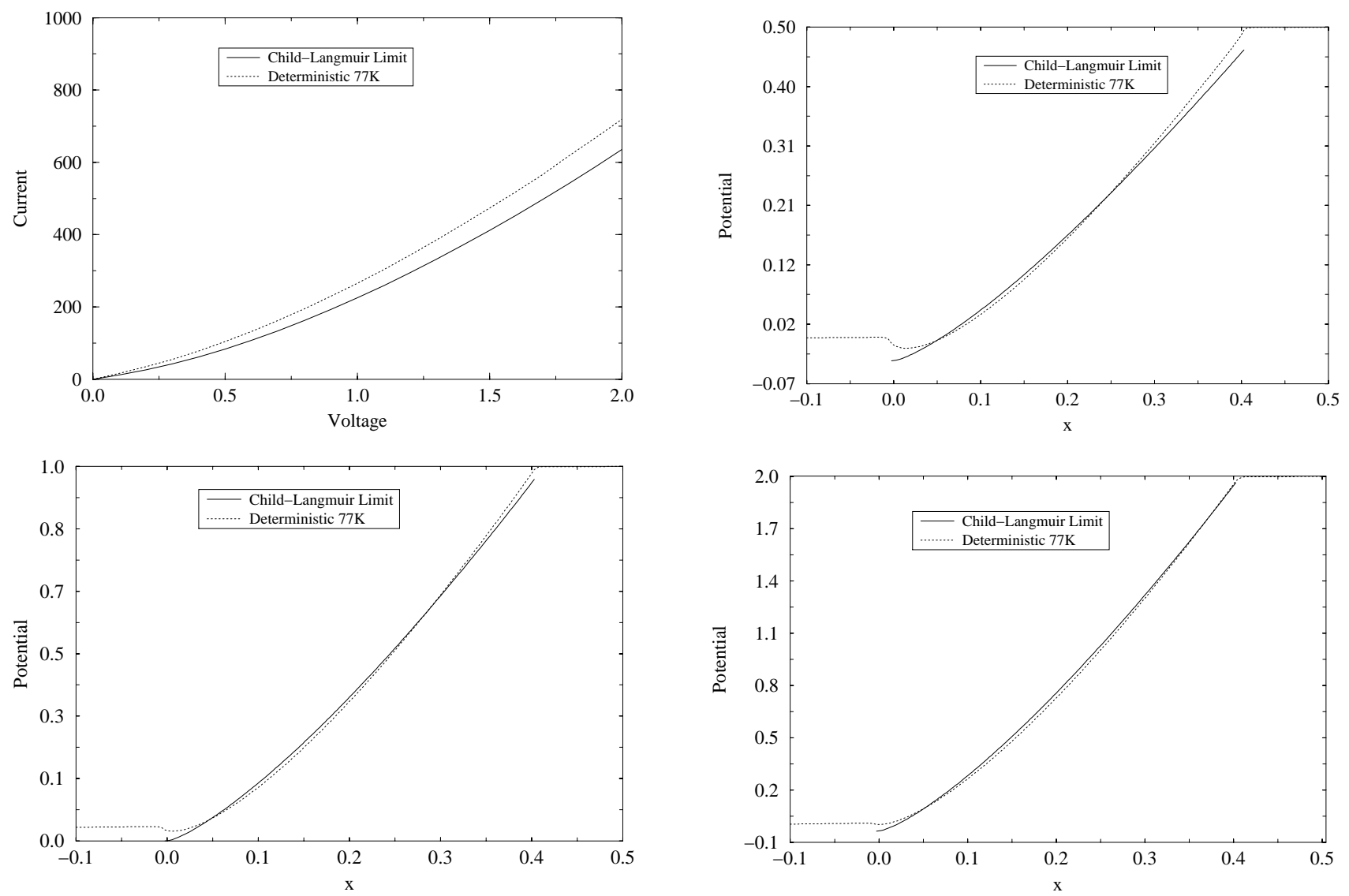

FiguRE 1. For Silicon device, at temperature $77 \mathrm{~K}$, numerical comparison of current-voltagecurves (top left) and potentials for three different $\phi_{L}$, namely, $0.5 \mathrm{~V}$ (top right), $1 \mathrm{~V}$ (bottom left), $2 \mathrm{~V}$ (bottom right). The influence of the built-in potential barrier into the numerical simulations for CL is taken into account by shifting the obtained profile by the corresponding value.

\subsubsection{Silicon device model}

For the Si device test case, the doping profile given by $C(x)$ is a sharp step function with density values $5 \times 10^{17} \mathrm{~cm}^{-3}$ in $-0.1 \leq x \leq 0$ and in $0.4 \leq x \leq 0.5$; and $2 \times 10^{15} \mathrm{~cm}^{-3}$ in $0<x<0.4$. In Table 3 , we give the standard parameters for $\mathrm{Si}$, where we recall the relation between $\mu$ and $\tau, \mu=\frac{e}{m} \tau$.

\subsubsection{GaAs device model}

For the GaAs device test problem, the doping profile given by $C(x)$ is a sharp step function with density values $10^{18} \mathrm{~cm}^{-3}$ in $-0.1 \leq x \leq 0$ and in $0.4 \leq x \leq 0.5$; and $2 \times 10^{15} \mathrm{~cm}^{-3}$ in $0<x<0.4$. In Table 4 , we provide the standard parameters considered for GaAs. 

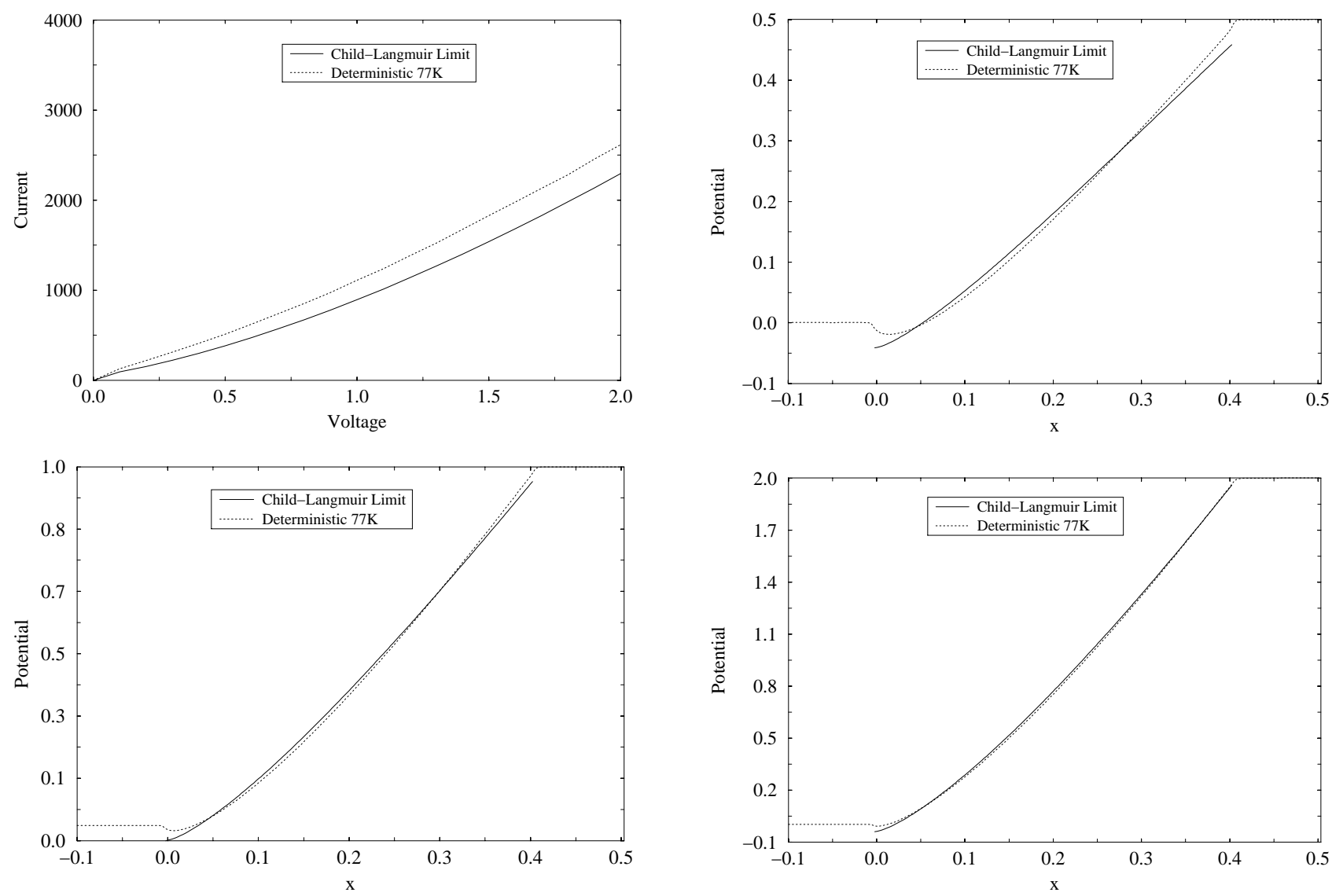

Figure 2. For GaAs device, at temperature $77 \mathrm{~K}$, numerical comparison of current-voltage characteristics curves (top left) and potentials for three different $\phi_{L}$, namely, $0.5 \mathrm{~V}$ (top right), $1 \mathrm{~V}$ (bottom left), $2 \mathrm{~V}$ (bottom right). The influence of the built-in potential barrier into the numerical simulations for CL is taken into account by shifting the obtained profile by the corresponding value.

\subsection{Numerical results}

\subsubsection{Current-voltage characteristics curves and potentials}

In Figures 1-2 we show comparisons between the current-voltage characteristics curves and the potentials for different applied biases.

Figure 1 is for Silicon device and Figure 2 is for GaAs device, both at temperature 77 K. For the CL limit, the built-in potential was taken into account, and thus, the agreement of the CL potential is closer to the potential of the kinetic equation in the central region of the channel. For both current-voltage characteristics curves and potentials, we can observe that simulations for the CL limit are better for large applied bias, $\phi_{L}$, in Si and GaAs devices. We show in Figure 3 the relative error for current-voltage characteristics curves, i.e.,

$$
E r=\frac{\left|J_{\mathrm{CL}}\left(\phi_{L}\right)-J_{\mathrm{D}}\left(\phi_{L}\right)\right|}{J_{\mathrm{D}}\left(\phi_{L}\right)}
$$

where $J_{\mathrm{CL}}\left(\phi_{L}\right)$ and $J_{\mathrm{D}}\left(\phi_{L}\right)$ denote the current given by numerical simulations for CL limit and deterministic system, with applied bias, $\phi_{L}$. 

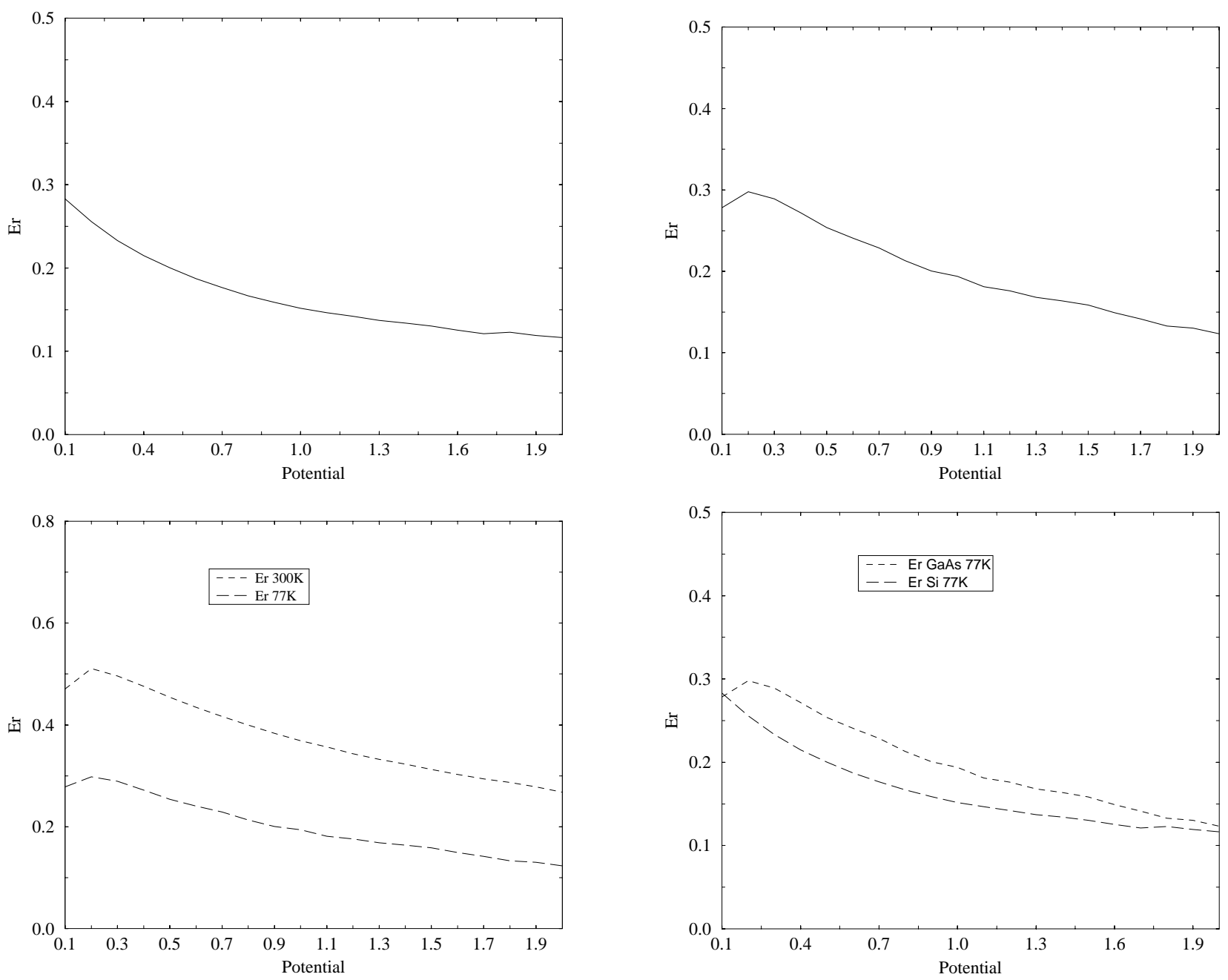

FiguRE 3. Relative error of CL Limit current-voltage characteristics curve: for Si at $77 \mathrm{~K}$ (top left), for GaAs at $77 \mathrm{~K}$ (top right), comparison for GaAs at $77 \mathrm{~K}$ and at $300 \mathrm{~K}$ (bottom left), comparison for $\mathrm{Si}$ and for GaAs at $77 \mathrm{~K}$ (bottom right).

Figure 3 also shows the relative error, Er, for the two different considered materials; Si and GaAs. In the bottom right figure, we provide a comparison between the relative error for $\mathrm{Si}$ and GaAs at temperature $77 \mathrm{~K}$, which shows that $E r$ for GaAs is larger than $E r$ for Si. However, for high potentials, relative errors for both materials are very similar. In this way, we could say that at temperature $77 \mathrm{~K}$, the numerical simulations for current-voltage characteristics curve for Si are more accurate than GaAs.

We recall that in the CL regime $m V_{\mathrm{th}}^{2} / 2 \ll e \phi_{L}$, therefore we can expect worse results at room temperature $(300 \mathrm{~K})$ than at $77 \mathrm{~K}$. In Figure 4 we consider, for the GaAs device, the current-voltage characteristics curve for CL limit and we compare it with the deterministic simulations at temperatures $77 \mathrm{~K}$ and $300 \mathrm{~K}$. Figure 3 shows the relative error for current-voltage characteristics curve for GaAs at temperatures $77 \mathrm{~K}$ and $300 \mathrm{~K}$, it proves that $E r$ is quite higher for $300 \mathrm{~K}$ than $77 \mathrm{~K}$, even for applied bias $\phi_{L}=2 \mathrm{~V}$. For the potentials, in the same way, the results are worse than at temperature $77 \mathrm{~K}$ although they improve when the applied potential is large. 

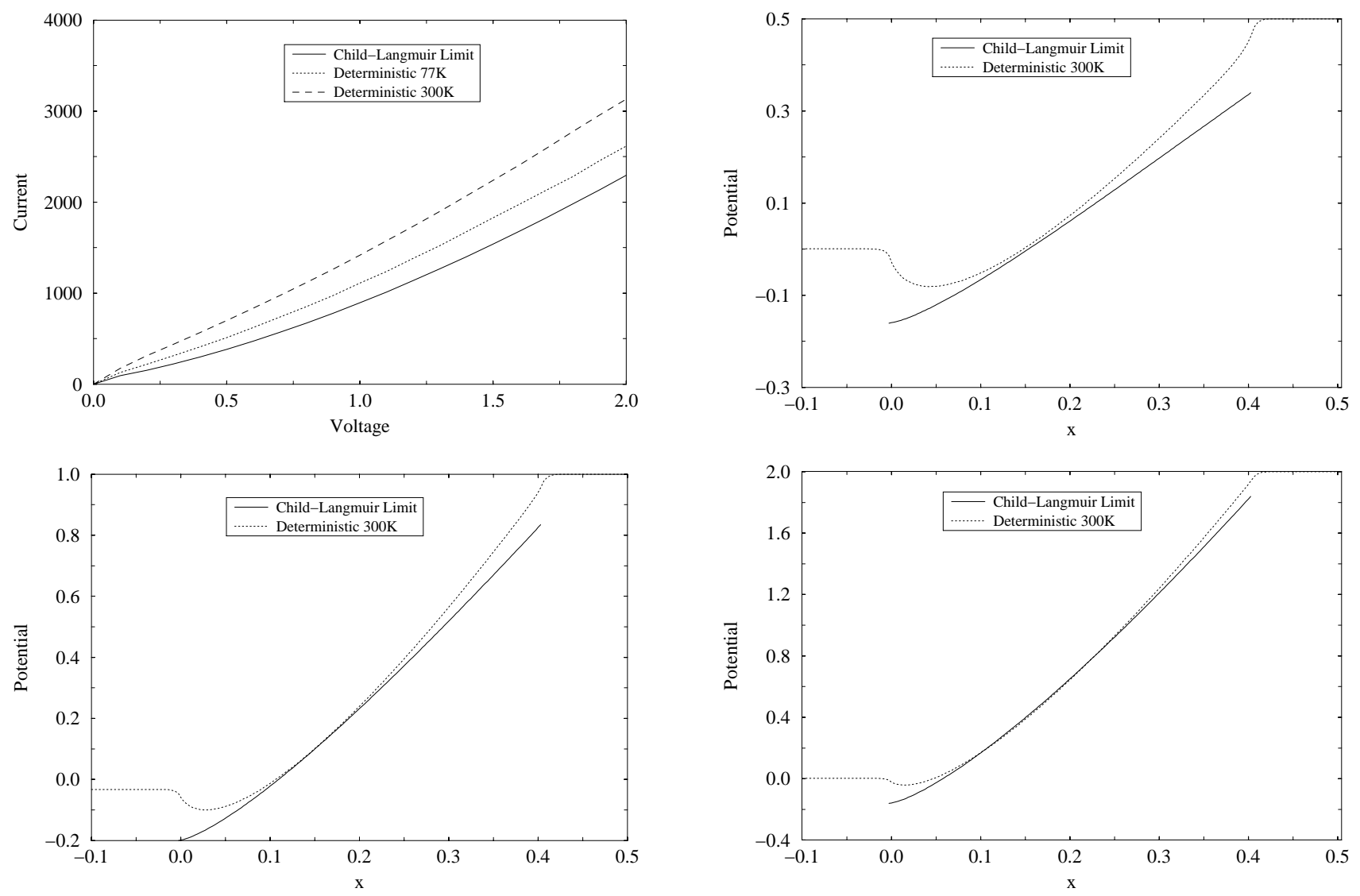

Figure 4. For GaAs device, at temperature $300 \mathrm{~K}$, numerical comparison of current-voltage characteristics curves (top left) and potentials for three different $\phi_{L}$, namely, $0.5 \mathrm{~V}$ (top right), $1 \mathrm{~V}$ (bottom left), $2 \mathrm{~V}$ (bottom right). The influence of the built-in potential barrier into the numerical simulations for CL is taken into account by shifting the obtained profile by the corresponding value.

\subsubsection{Charge densities and distribution functions}

In Figures 5-6 we show the charge density for an applied potential $\phi_{L}=1 \mathrm{~V}$ at temperatures $77 \mathrm{~K}$ and $300 \mathrm{~K}$. In these figures we can observe that the CL regime is quite right for the central zone of the channel and it is, even, better for the end of the central zone. On other hand, in these Figures, we can check again that, the CL asymptotic limit is better for low temperatures.

Figures $7-8$ show the normalized stationary distribution at temperatures $77 \mathrm{~K}$ and $300 \mathrm{~K}$, at $x=0.18 \mu \mathrm{m}$ and $x=0.34 \mu \mathrm{m}$, for Silicon device. At these points we can expect sharper results, since the more accurate results for the density are given for the interval $[0.15 \mu \mathrm{m}, 0.35 \mu \mathrm{m}]$ (see Fig. 5). These computations are performed by replacing the Dirac delta by $\frac{1}{\epsilon} M_{0}\left(\frac{v}{\epsilon}\right)$, taking $\epsilon=\frac{V_{\mathrm{th}}}{V_{L}}$ or equivalently $\epsilon=\sqrt{k_{\mathrm{B}} T / 2 e \phi_{L}}$. Table 1 shows $\epsilon$ for different temperatures and applied biases, which were used in Figures $7-8$. We can check that, for temperature $300 \mathrm{~K}, \epsilon$ is large, therefore we can expect the results of the CL are not so accurate. The value of $\epsilon$ at $300 \mathrm{~K}$ and $\phi_{L}=2 \mathrm{~V}$ is very close to the value of $\epsilon$ at temperature $77 \mathrm{~K}$ and applied bias $0.5 \mathrm{~V}$.

In Figures 7-8 we observe that the distribution functions present two peaks, according to the thermal speed and the ballistic velocity $\sqrt{2 e \phi(X) / m}$. Both peaks are captured by the CL limit. Although, the peak corresponding to the ballistic speed is better captured. Therefore, it is better when the ballistic velocity is large, 

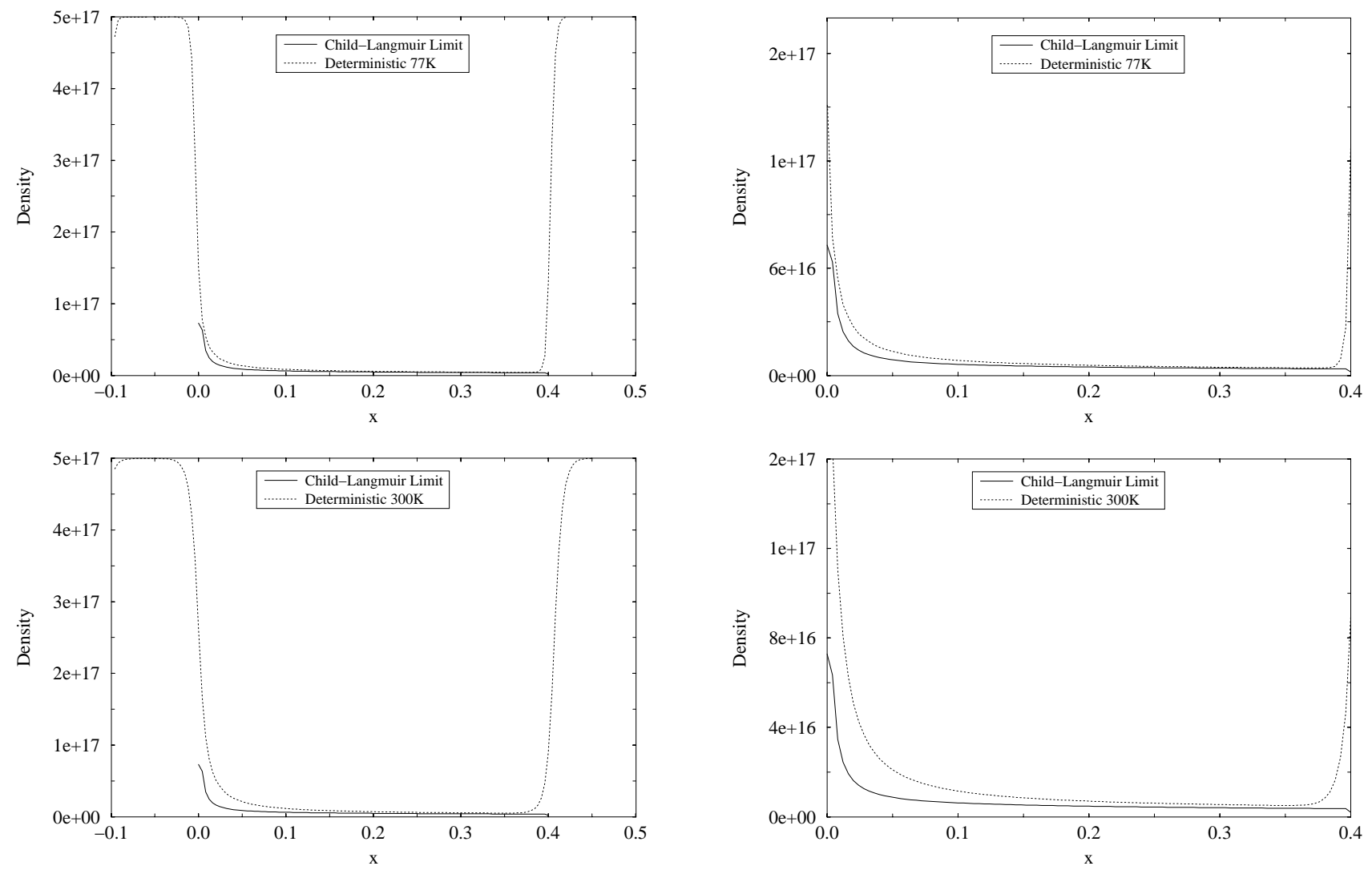

FIGURE 5. For Silicon with applied potential 1 V. Numerical comparison of the charge densities at temperature $77 \mathrm{~K}$ (top) and at temperature $300 \mathrm{~K}$ (bottom).

namely, for large potential, (we can compare, in Fig. 7, the results for $\phi_{L}=0.5 \mathrm{~V}$ and $\phi_{L}=2 \mathrm{~V}$ ). Again, comparing the results for different temperatures, we appreciate that simulations for temperature at 300 K (Fig. 8) are not as good as for $77 \mathrm{~K}$ (Fig. 7).

In Figure 9 we show the normalized distribution for GaAs device, at temperature $77 \mathrm{~K}$ at points $x=0.18 \mu \mathrm{m}$ and $x=0.34 \mu \mathrm{m}$, for two different applied potential, $\phi_{L}=0.5 \mathrm{~V}$ and $\phi_{L}=1 \mathrm{~V}$. Again we use the maxwellian to approximate the delta function (see Tab. 1 for the value of $\epsilon$ ). For GaAs, the ballistic velocity is larger, therefore the ballistic peak is more important than the peak for the thermal speed. The CL captures the ballistic peak and at $\phi_{L}=1 \mathrm{~V}$ we can observe, also, that CL "tries to capture the thermal tail". Therefore, we can say that transport in GaAs is more ballistic than $\mathrm{Si}$.

\subsubsection{Moments}

In Figures 10 and 11 we study the first three moments of the distribution function, showing: the mean velocity, total energy, mean temperature and third moment, i.e.,

$$
u=\frac{J}{\rho(F)}, \quad \frac{\int_{\mathbb{R}^{3}}|V|^{2} F \mathrm{~d} V}{2 \rho(F)} m+e \phi(X), \quad \frac{\int_{\mathbb{R}^{3}}(V-u)^{2} F \mathrm{~d} V}{e \rho(F)} m, \text { and } \int_{\mathbb{R}^{3}} V^{3} F \mathrm{~d} V .
$$

In Figures 10-11 we compare moments of the deterministic simulation with two possible numerical approximations for the moments of the CL limit: first, approximating the CL distribution (2.10) by replacing $\delta(v)$ with the maxwellian of variance $\epsilon^{2}$, Child-Langmuir $\epsilon$; second, direct evaluation of the moments by operating 

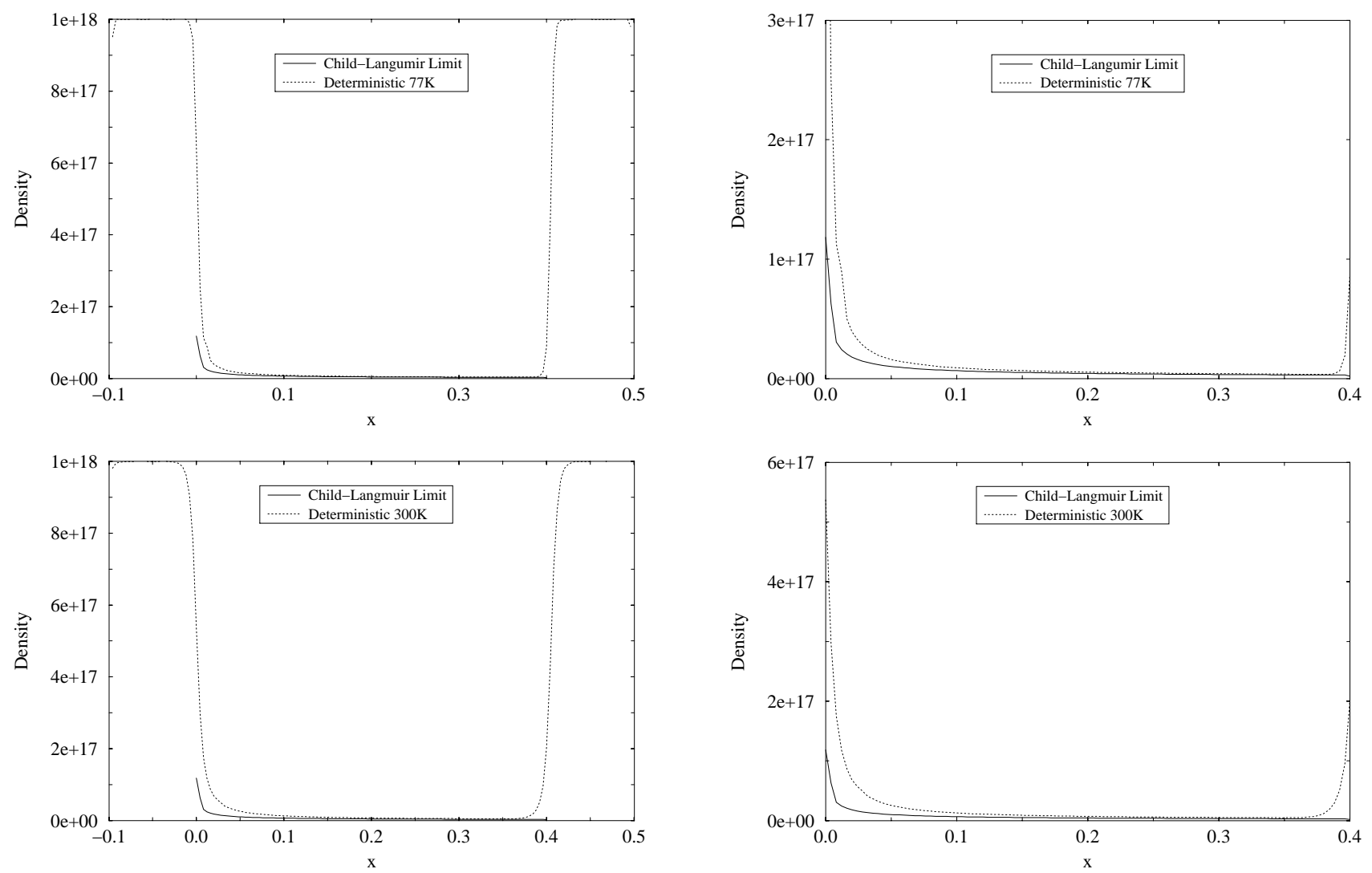

Figure 6. For GaAs with applied potential $1 \mathrm{~V}$. Numerical comparison of the charge densities at temperature $77 \mathrm{~K}$ (top) and at temperature $300 \mathrm{~K}$ (bottom).

over (2.10), Child-Langmuir Limit. The last moment approximation is the real numerical approximation for CL limit, since we take into account the limit distribution function (2.10).

For both, Si and GaAs, the temperature was $77 \mathrm{~K}$ and $\phi_{L}=1 \mathrm{~V}$. The figures show, that in general, $C L \epsilon$ is more accurate than $C L$ Limit except for the total energy. Since the potential energy is the same for $C L \epsilon$ and CL Limit, therefore we could expect similar results for the total energy.

In the case of mean temperature, $C L \epsilon$ is better than $C L$ Limit for $\mathrm{Si}$, while for GaAs the opposite happens, namely, $C L$ Limit approximation is better than $C L \epsilon$.

The third moment for GaAs shows a quite bad approximation for $C L$ Limit. However, $C L \epsilon$ gives a more precise estimate. For Si this large difference between $C L$ Limit and $C L \epsilon$ disappears.

\section{Conclusions}

A numerical validation of the Child-Langmuir asymptotic limit for the 1-D constant relaxation time kinetic system is given by performing detailed numerical comparisons between the simulation of the Boltzmann-Poisson system and the CL asymptotic equations for $\mathrm{Si}$ and $\mathrm{GaAs} \mathrm{N}^{+}-\mathrm{N}^{-}-\mathrm{N}^{+}$diodes.

For our simulations we consider the same device for two different materials: Si and GaAs. We check that at $77 \mathrm{~K}$ the CL limit current-voltage characteristics curve for Si is better than for GaAs, although, for large applied biases, results for both materials tend to be similar. Although, at $300 \mathrm{~K}$ the results are worse for Si than for GaAs. 

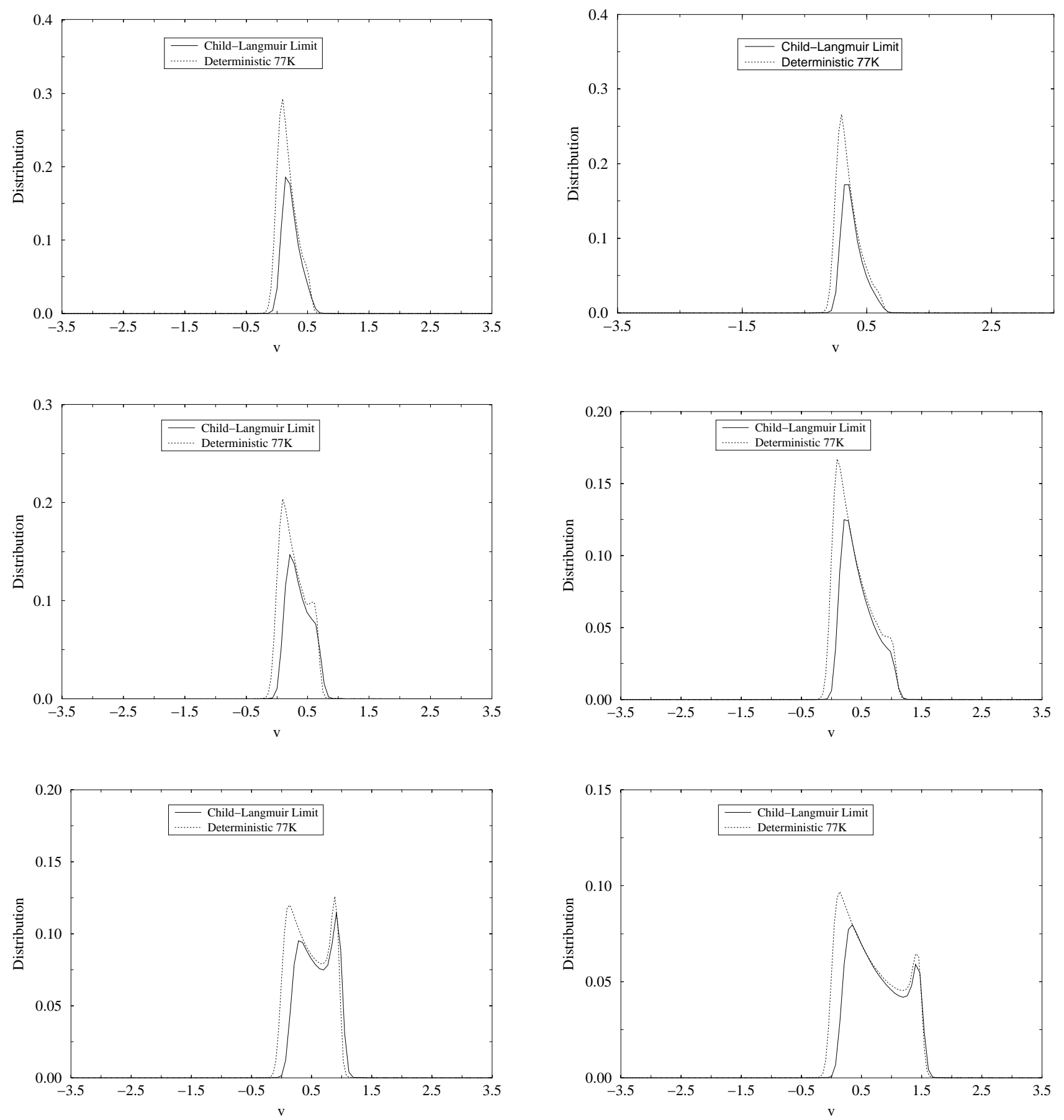

FiguRE 7. For Silicon, at temperature $77 \mathrm{~K}$, numerical comparison of normalized stationary distributions, at several fixed $x$ for different applied potentials: $0.5 \mathrm{~V}$; at $x=0.18 \mu \mathrm{m}$ (left top) and at $x=0.34 \mu \mathrm{m}$ (right top), $1 \mathrm{~V}$; at $x=0.18 \mu \mathrm{m}$ (left middle) and at $x=0.34 \mu \mathrm{m}$ (right middle) and $2 \mathrm{~V}$; at $x=0.18 \mu \mathrm{m}$ (left bottom) and at $x=0.34 \mu \mathrm{m}$ (right bottom). 

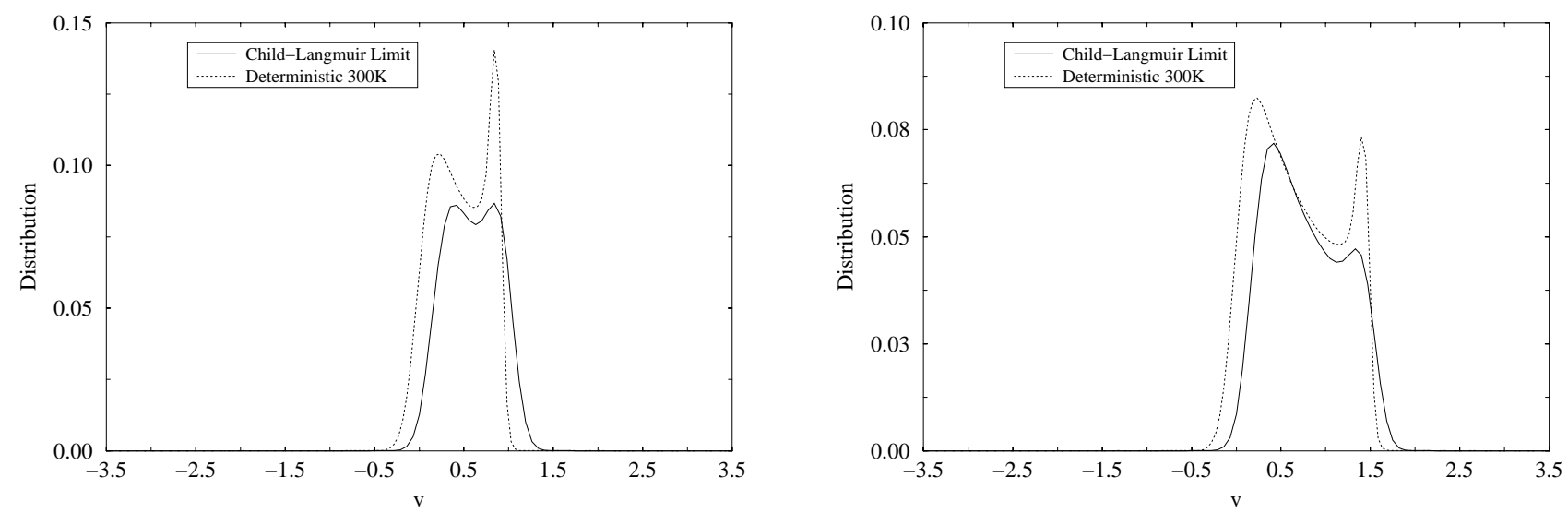

Figure 8. For Silicon, at temperature $300 \mathrm{~K}$, numerical comparison of normalized stationary distributions for $\phi_{L}=2 \mathrm{~V}$ at $x=0.18 \mu \mathrm{m}$ (left) and at $x=0.34 \mu \mathrm{m}$ (right).
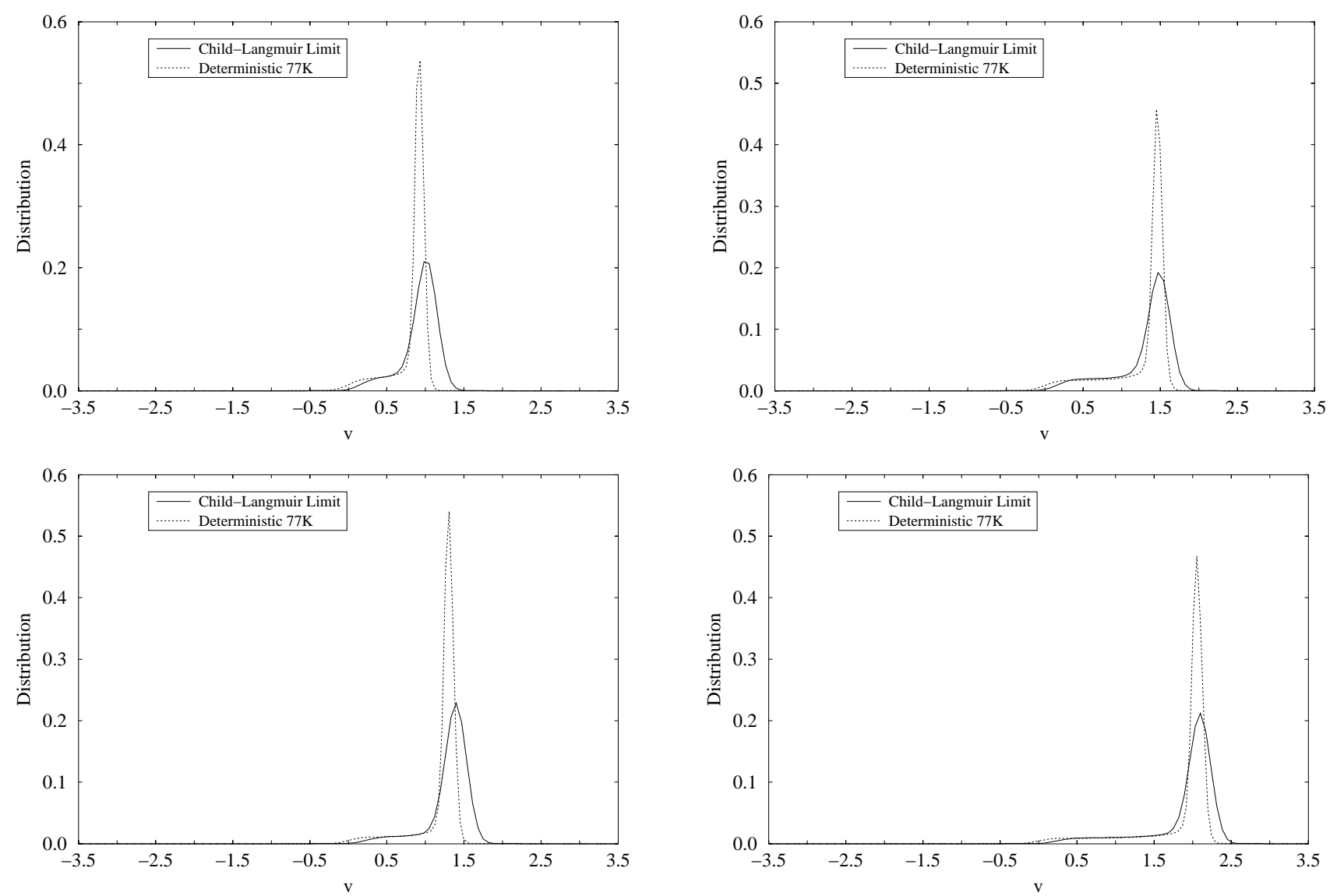

Figure 9. For GaAs, at temperature $77 \mathrm{~K}$, numerical comparison of normalized stationary distributions, at several fixed $x$ for different applied potentials: $0.5 \mathrm{~V}$; at $x=0.18 \mu \mathrm{m}$ (left top) and at $x=0.34 \mu \mathrm{m}$ (right top) and $1 \mathrm{~V}$; at $x=0.18 \mu \mathrm{m}$ (left bottom) and at $x=0.34 \mu \mathrm{m}$ (right bottom). 

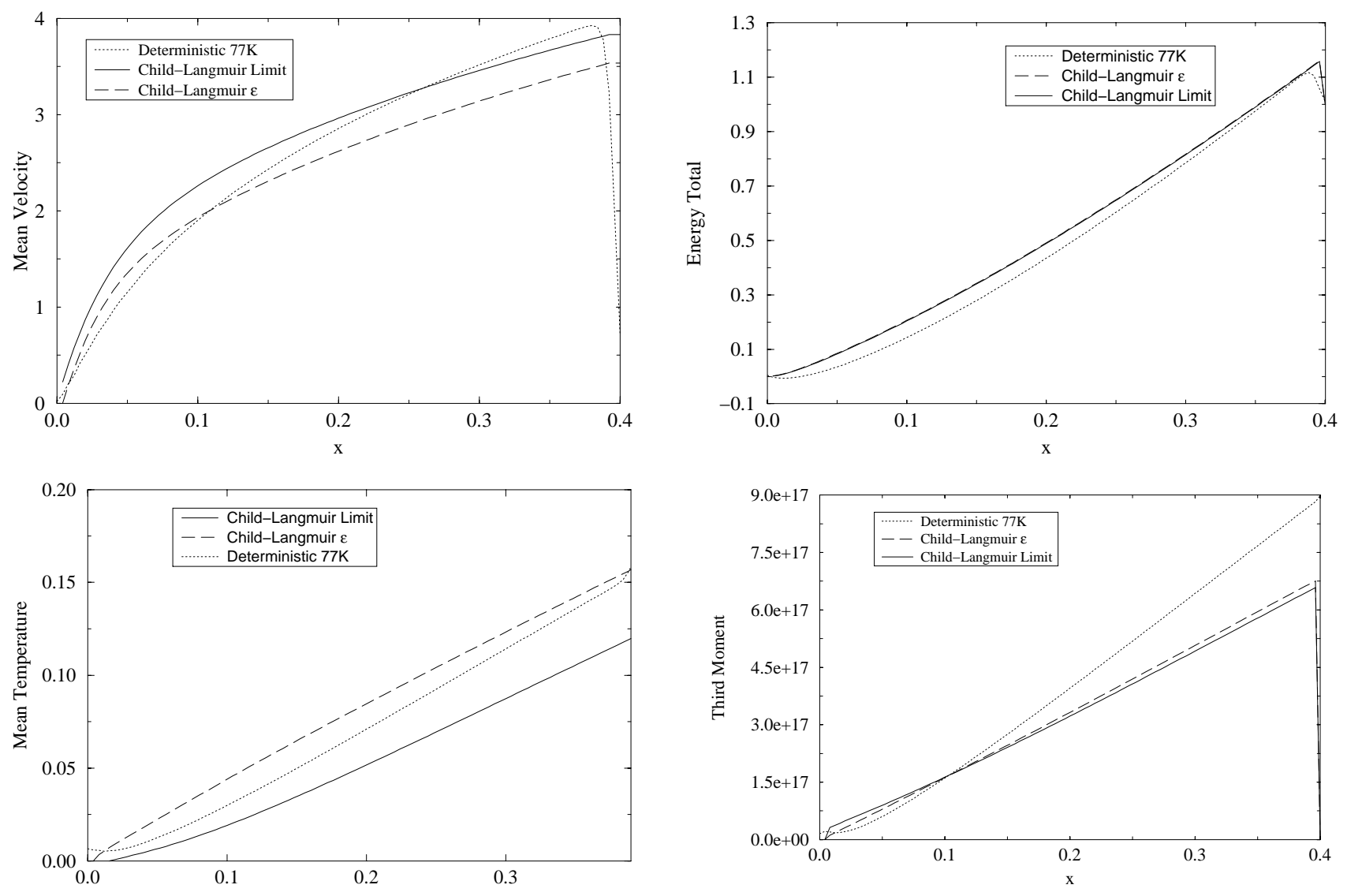

Figure 10. For Silicon, at temperature $77 \mathrm{~K}$ and applied potential $\phi_{L}=1 \mathrm{~V}$, numerical comparison of mean velocity (left top), total energy (right top), mean temperature (left bottom) and third moment (right bottom).

The numerical simulations for the densities show that the most accurate results are obtained at the center of the channel. In this way, at $x=0.18 \mu \mathrm{m}$ and $x=0.34 \mu \mathrm{m}$, the normalized distribution functions capture the peak corresponding to the ballistic and thermal speeds (we must recall that the Child-Langmuir scaling is a ballistic scaling, Rem. 2.1). Also, our simulations show that the ballistic peak is better captured than thermal one. Finally, we have compared moments of the distribution obtained through the CL limiting solution with deterministic simulations. It has been shown that, in general, better results are obtained by restituting a finite temperature feature in the CL limit solution.

It is worthy to point out that, following our study, simulations of the CL limit can be very useful for providing initial guesses for the current-voltage characteristics curves and for the solution of the stationary BoltzmannPoisson system via (2.10) with a very low computational cost. In fact, computer time for the Child-Langmuir Limit is negligible (almost instantaneous) compared to the computational time for the relaxation time operator (about 20 minutes); our simulations were carried out on a Pentium II $500 \mathrm{MHz}$. For instance, the CL limiting distribution function can be taken as a more accurate initial data in direct schemes for solving the stationary Boltzmann-Poisson system, eventually accelerating them. On the other hand, the CL limiting results can be considered as a quick tool for computing current-voltage characteristics if we allow ourselves a relative error according with Figure 3. Let us remark that the CL limit is valid for situations (low temperature, high bias) in which other approximations, as drift-diffusion or hydrodynamic simulations, may not be well suited. 

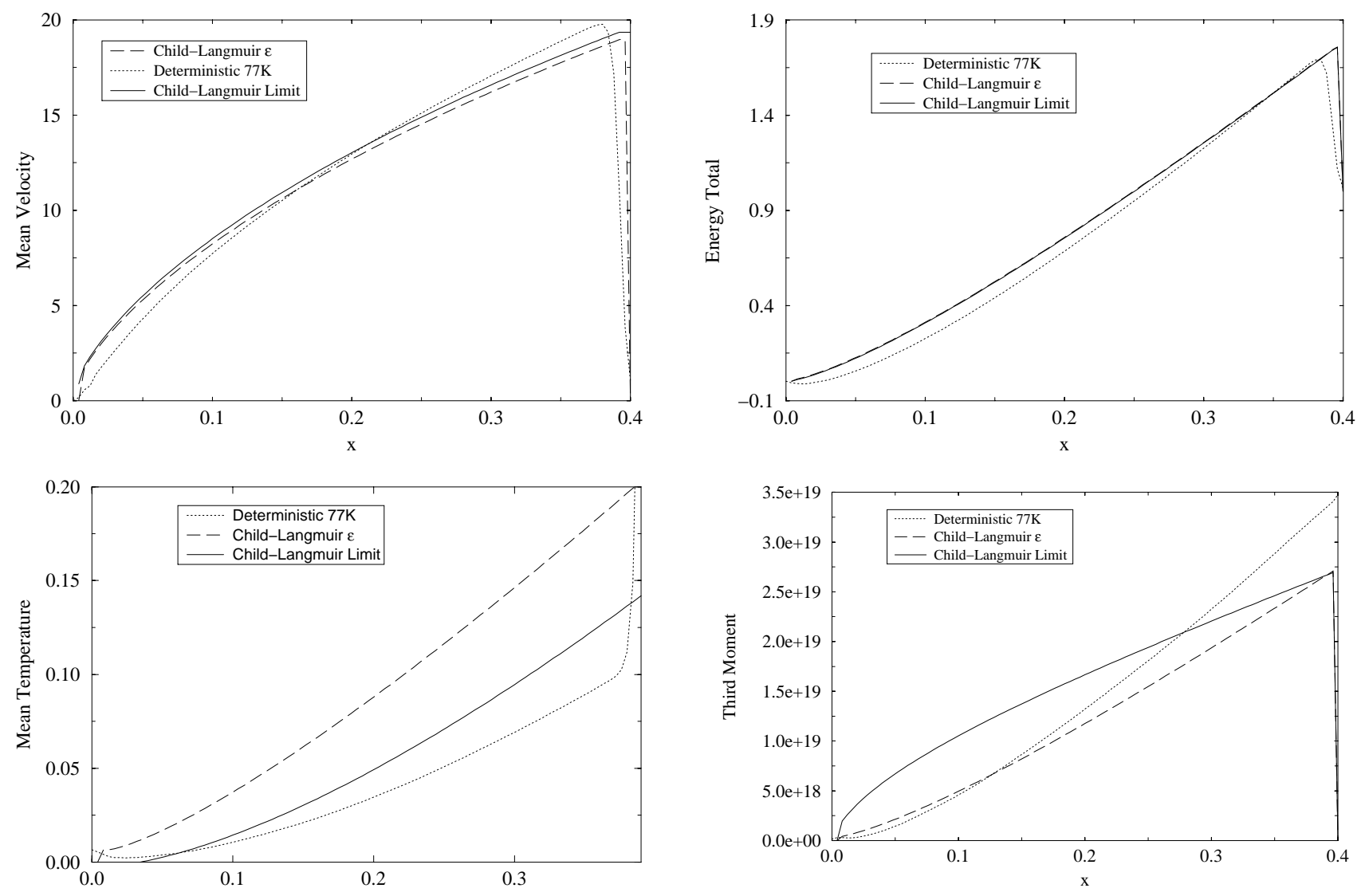

Figure 11. For GaAs, at temperature $77 \mathrm{~K}$ and applied potential $\phi_{L}=1 \mathrm{~V}$, numerical comparison of mean velocity (left top), total energy (right top), mean temperature (left bottom) and third moment (right bottom).

We can conclude noting that the numerical simulations are in accordance with expected results, if one bears in mind that the Child-Langmuir law gets better as the applied bias gets higher or as the lattice temperature gets lower.

Acknowledgements. The authors would like to warmly thank Prof. Ben Abdallah for making his code for the CL solution available to them and for sharing with them his experience on it. The authors were partially supported by the European IHP network "Hyperbolic and Kinetic Equations: Asymptotics, Numerics, Applications", RNT2 2001 349. M.J.C. and J.A.C. acknowledge support from DGI-MCYT project BFM2002-01710.

\section{REFERENCES}

[1] F. Alabau, K. Hamdache and Y.J. Peng, Asymptotic analysis of the transient Vlasov-Poisson system for a plane diode. Asymptot. Anal. 16 (1998) 25-48.

[2] H.U. Baranger and J.W. Wilkins, Ballistic structure in the electron distribution function of small semiconducting structures: General features and specific trends. Phys. Rev. B 36 (1987) 1487-1502.

[3] N. Ben Abdallah, The Child-Langmuir regime for electron transport in a plasma including a background of positive ions. Math. Models Methods Appl. Sci. 4 (1994) 409-438.

[4] N. Ben Abdallah, Convergence of the Child-Langmuir asymptotics of the Boltzmann equation of semiconductors. SIAM J. Math. Anal. 27 (1996) 92-109. 
[5] N. Ben Abdallah, Étude de modèles asymptotiques de transport de particules chargées: Asymptotique de Child-Langmuir. Ph.D. thesis.

[6] N. Ben Abdallah and P. Degond, The Child-Langmuir law for the Boltzmann equation of semiconductors. SIAM J. Math. Anal. 26 (1995) 364-398.

[7] N. Ben Abdallah and P. Degond, The Child-Langmuir law in the kinetic theory of charged particles: semiconductors models. Mathematical problems in semiconductor physics, Rome (1993) 76-102. Longman, Harlow, Pitman Res. Notes Math. Ser. 340 (1995).

[8] N. Ben Abdallah, P. Degond and F. Méhats, The Child-Langmuir asymptotics for magnetized flows. Asymptot. Anal. 20 (1999) 97-13.

[9] N. Ben Abdallah, P. Degond and C. Schmeiser, On a mathemaical model of hot-carrier injection in semiconductors. Math. Methods Appl. Sci. 17 (1994) 1193-1212.

[10] J.A. Carrillo, I.M. Gamba, O. Muscato and C.-W. Shu, Comparison of Monte Carlo and deterministic simulations of a silicon diode. IMA series (to be published).

[11] J.A. Carrillo, I.M. Gamba and C.-W. Shu, Computational macroscopic approximations to the 1-D relaxation-time kinetic system for semiconductors. Phys. D 146 (2000) 289-306.

[12] P. Degond and P.A. Raviart, An asymptotic analysis of the one-dimensional Vlasov-Poisson system: the Child-Langmuir law. Asymptot. Anal. 4 (1991) 187-214.

[13] P. Degond and P.A. Raviart, On a penalization of the Child-Langmuir emission condition for the one-dimensional VlasovPoisson equation. Asymptot. Anal. 6 (1992) 1-27.

[14] G. Jiang and C.-W. Shu, Efficient implementation of weighted ENO schemes. J. Comput. Phys. 126 (1996) 202-228.

[15] I. Langmuir and K.T. Compton, Electrical discharges in gases: Part II, fundamental phenomena in electrical discharges. Rev. Modern Phys. 3 (1931) 191-257.

[16] P.A. Markowich, C.A. Ringhofer and C. Schmeiser, Semiconductor Equations. Springer, New York (1990).

[17] C.-W. Shu, Essentially non-oscillatory and weighted essentially non-oscillatory schemes for hyperbolic conservation laws, Advanced Numerical Approximation of Nonlinear Hyperbolic Equations, B. Cockburn, C. Johnson, C.-W. Shu and E. Tadmor (A. Quarteroni Ed.). Springer, Lecture Notes in Math. 1697 (1998) 325-432.

[18] M.S. Shur and L.F. Eastman, Ballistic transport in semiconductors at low temperature for low-power high-speed logic. IEEE Trans. Electron Dev. ED-26 (1979) 1677-1683.

[19] M.S. Shur and L.F. Eastman, Near ballistic transport in GaAs devices at 77 K. Solid-State Electron 24 (1991) 11-18.

To access this journal online:

www.edpsciences.org 